\title{
Landscape Change and Human Environment
}

\author{
Asad Aziz , Muhammad Mushahid Anwar \\ Department of Geography, University of Gujrat, Gujrat., Village Rehsian.P/O Kotla Arab Ali khan \\ Tehsil Kharian Distt Gujrat., 50990 Kotla arab ali khan, Pakistan
}

Received: 28 Apruil 2018

Accepted: 30 June 2019

Published online: 30 December 2019

\begin{abstract}
Landscape and human are the constituents of the natural surroundings in which changing occurred temporally and spatially. The relationship between landscape and environment is not seen by a singular framework or a bare structure. A landscape has different functions and characteristics, when meet specific requirements, then set an ecology in giving context. Every landscape has its own scenario based upon the dimension of human and its kinship with them. Man are the ecosystem managers, the landscape features provide a road map and built human ecology. Development is occurring by landscape change, change of environment and the kinship between human and landscape, study of these phenomena provide us a theoretical framework to assess the man land relationship as is done in this case study. This paper aims to describe how, each individual in a particular environment is participating in change of the landscape. The influence of human is a magnitude of actions upon the landscape and this magnitude shape the local landscape. The paper discussed through a brightness on the dynamics of landscape change in the 21st century due to globalization and modern dimensions and presents us a complex web of human with non-human relationship of ecology, economy and history, which alter the landscape. This paper concluded a reflection of cultural values on physical environment and landscape change to build environment and human ecology.
\end{abstract}

Keywords: Human ecology, human activities, environment, landscape, Landscape Changes

\section{Introduction}

Landscape a mosaic of interacting ecosystem perceptually or an expense which is heterogeneous in parameter of interest. This term have different mechanisms in different discipline including geology, geography, art, history politics, planning and design, although all of these features are physical but in current era the landscape change are just a scenery (Kaymaz 2012). In 21st century a number of critical changes drive variety of environmental problems which not only effect the local landscape but also the whole world, the landscape changes display a resolute image of environmental crises (Çelik 2013). The environment provides all of functions and services which are driving tools for the life, men modified the landscape in more than one way to fulfill the needs in everyday life. Human are the actors and landscape are symbolic representation on nature and environment (Garkovich 1994). Placelessness due to globalization and modernity trends to build the human environment and cause

${ }^{*}$ Corresponding author: Asad Aziz

e-mail: asadazizpakistan@gmail.com 
destruction in natural landscape. The interaction between given environment and landscape provide a phenomenological relationship of human ecology (El-Shafie 2010). Irreversible and unacceptable environmental change are the challenges facing by the society today (Clive et al. 2013).

The social is man-made and physical is natural, both create different types of structures by which the particular population has different synchronization, that based upon their functions and create an environmental relationship naturally. The pattern of landscape, their history, and historical ecology provide us a way to understand the ecology of men and built environment (Erickson 2010). The nature and society are unpredictable and dynamic phenomena's at any place on earth surface. Everything in environment is related to each other in evident or secreted meaning (Burgi 2004). Water flow from high surface to low surface, a mountain eroded slowly due to weather actions and people moved from one location to another location. Better the landscape change management more the effective way of urban services to be used ( Belanche 2016). The cultural context also has its impact on landscape in a given area based upon local cultural values. Transformations of natural landscape into cultural landscape give a real context of human approaches. Landscape planning is forceful tool for sustainable development.

Environmental and ecology, study the interdependence and interrelationships between nature and environment functionally and thematically and also constitute primary drivers for ecological studies (Bekoff 2000).

The social environment is judge qualitatively, while the economical and physical environment is judge by the quantifications (Burgi 2004). Now a day the landscapes changes are accelerated by a greater magnitude by the cultural changes. The cultural changes and changes in landscapes is a journey of undefined goals (Burgi 2004).

The cultural changes have different forms, may be technology, economy, population change, education or political forces (Burgi 2004). The humans always bring changes in their environment by different events, in which more dominant are economic (Aretano 2013). The local, administrative, political, cultural and population change brings great changes in landscape and nature with space and time(Aretano 2013).

Land and other components of ecosystem are finite and their demand is goes on increasing and create a conflict between land users ("Landscapes of the Future" 2011). The nature of landscape changes depends upon the natures of population lived in a regions theirs activates and functions performed by them (Aretano 2013). Landscape management is very important for developmental strategies and environmental management (Belanche 2016). Greater the time more the landscape changes more activates occurred, so more dominant on the landscape the humans are. The idea of landscape change is taking from landscape, where nature and culture are meeting. The investigation of these phenomena gives us a brief understand about environmental crises (Arntzen 1982).

The socioeconomic is the base of economy and directly interlinked with political laws and policies the new emerged driving forces is technology, have great and fast impact on changing landscape, main while the site factors like climate, topography, soil characteristics and major distributed factors in land escape changes (Burgi 2004). These factors required a greater time to change the landscape while the fast actors which bring the changes in landscape shortly are, avalanches, mudslides, and hurricanes ( Burgi 2004).

Every environmental factor have its own affect, a cultural impact involved the cultural changes while the natural factor involved the natural changes. The relationship between cultural and environment is still a great vague however it can be identified by the time and space variation and required a complex method of investigation. The time ranging from months to years and decades, spatial variations including individual to states, states to continents around the world (Burgi 2004). Deep and strong effects can be seen on landscape and environment by the construction if human niche, 
Human are managers of ecosystem which brings the changes in landscape and environment (Fuentes and Baynes-Rock 2017).

The road networking, streets and pipelines of urban services are also consider as the tool for changing the urban environment (Belanche 2016). Underground tunnels, road infrastructure and urban settlements cause to change the landscape locally as well as globally.

The change of landscape is not a result of intentional actions but it's also due to planned actions, urbanization and globalization are the major process, causes great changes in landscape. It's due to happening of unexpected effects sides. It is a difficult task to differentiate between change of accidental landscape and their related driving forces, every organism in a given community is involved in the process of evolution (Steiner 2017).

\section{Methods}

The method involved in case study is not fit into a perfect framework it is a problem oriented as it is not restricted into a specific practice. The relationship of peoples lived in a particular region with time and space, its analysis and study gives us a comprehensives data about landscape change and ecology of local residents.

\section{Intuitional Resolution _-- Time Resolution _ -- Landscape Resolution}

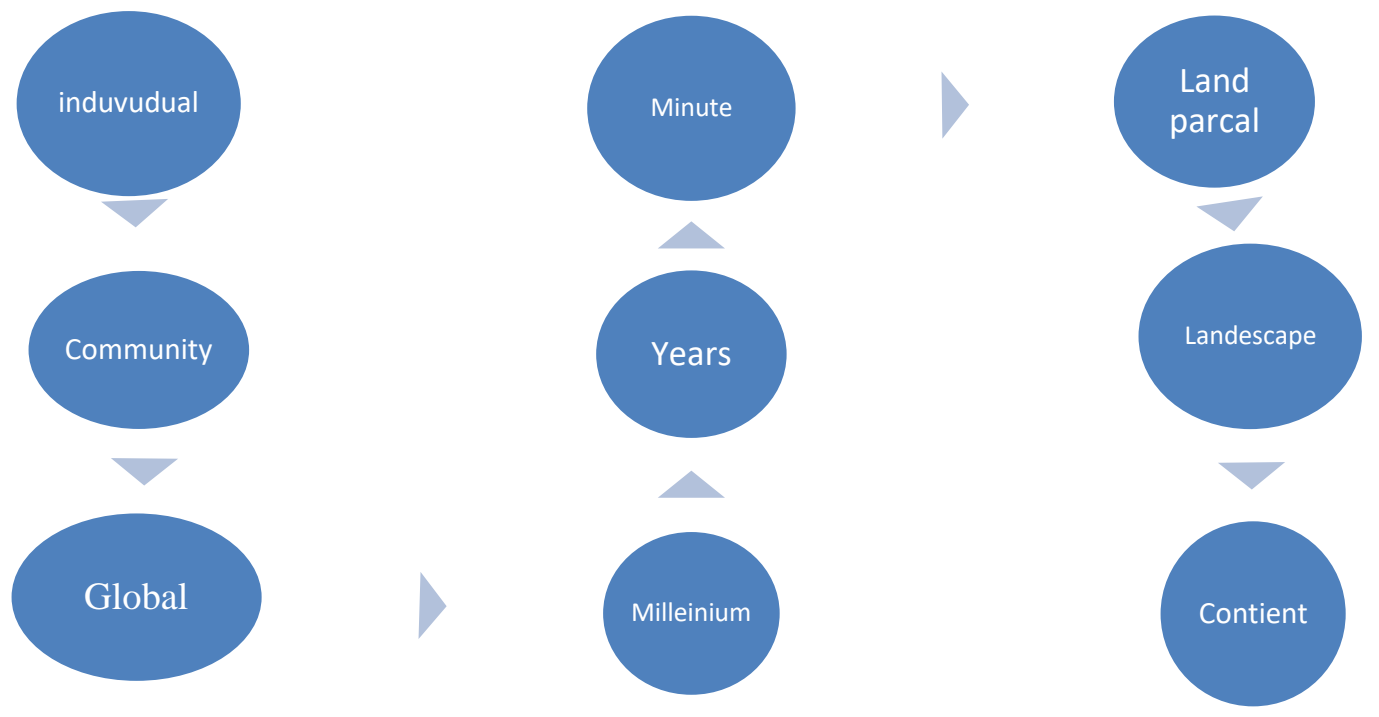

Fig. 1. Framework landscape change, temporal and spatial changes.

The simple system describes the components of landscape change with spatial and spatial resolution give us a brief methodological chain involved in landscaping changing process, and actors involved in landscaping changing. Functional components involved and forms the landscape system. The system formed by the landscape is very complex and this complex system in made up by the actors acting in landscape, drive different task and then change it with time to time greater the actions or more the actors, more the time, the greater the extent of landscape. The analysis of driving forces like culture, technology, socioeconomic and nature gives a lot of information about the environment and landscape changing. Greater the land use greater the landscape changes because the human modified the landscape to build the environment according to their needs (Belanche 2016). 
Landscape are represented by different classes "urban or natural" (Velardea 2007). Landscape changes occurred by the change of environment physically or socially of any regions locally as well as globally, acid rain, global warming, erosion, soil salinity, increase of population, urban sprawl, droughts, floods, extreme water and climate change, cultural phenomena's including urban settlement, road networking or commercial land use. Implementation of these changes, may be natural or synthetic, accelerate the landscape change and drive the tool to fast-track the environmental changes may be visible or underlying. The time and scale determined the level of examinations and resolution of changes. The landscapes are merge or changed by different activities of human and this is very complex matter to understand the ecosystem. Landscape are also a part of environment, so there is a reciprocal relationship between human and environment (Parsaee 2015).

An individual act on land and change its structure in short time as the scale is small may be a minute in temporal scale, while if we expand our thinking at global level, then the landscape of a continent may be changed by changing the actors because a more population is involved, the nature of driving forces involved in changing and factors which cause the changing so greater time is required to bring it, may be Millenniums. All of these elements are correlated with each other and the use of statistical methods gives us a strong picture of correlation between them.

\section{Discussion}

A landscape a heterogeneous area in size, shape, characteristics and natural phenomena's throughout the region. The every species in given or specific population or a particular individual in a population in involved and play its individual role in changing the environment by changing the structure of the landscape. It may be a human, an animal or a plant. Evolution in landscape is done and accelerated by the involvement of resident living in that area, the human adopted their local environment locally and modified it according to theirs need, in some cases and times the human is dominated in the environment may be called as possibilism while the response of environment opposite to it, called determinism. The process by which a landscape changed are economic activities, cultural process, developmental actions and migration in both humans and animals, natural phenomena's and human social activities. A landscape have different components including work areas, public area for services, children parks, and private use area and fronts yards (Riley 2015).

The ecological demands create trends to bring changes in natural landscape, the biodiversity is deceased and settlement is established, a forest is replaced by urban elements. So being the part of this earth surface we all of us the element of ecosystem and involved with the environment in different forms, this is concluded in this Paper.

The accelerators are the challenges which bring or cause the changing in nature, landscape and environment. Socioeconomic, political, technological, natural and cultural forces which were adopted by the human and modified the environment and ecosystem. The landscape gives us a way for decisions making, policy making and human adoptions with culture and nature. Land usage have a contributed effects on landscape change shaping the environment (Zube 1987).

Landscape changing is a continuous process in any environment depends upon its location and different events action on it. Ecology, land use pattern and social behavior are important components to determine the cultural ecology and also key tools for the frame work of research. Nature have its orderly version in forms of landscape design and in this designing the natural landscape is removed by installing the new settlements (Langellotto-Rhodaback 2015).

The human as the actors as described earlier, when act to bring changes in the given landscape and environment to meet their needs. The needs and landscape change combined to built ecology, the given environment and natural landscape meets it. But all of these have only a single source that is ecosystem. 


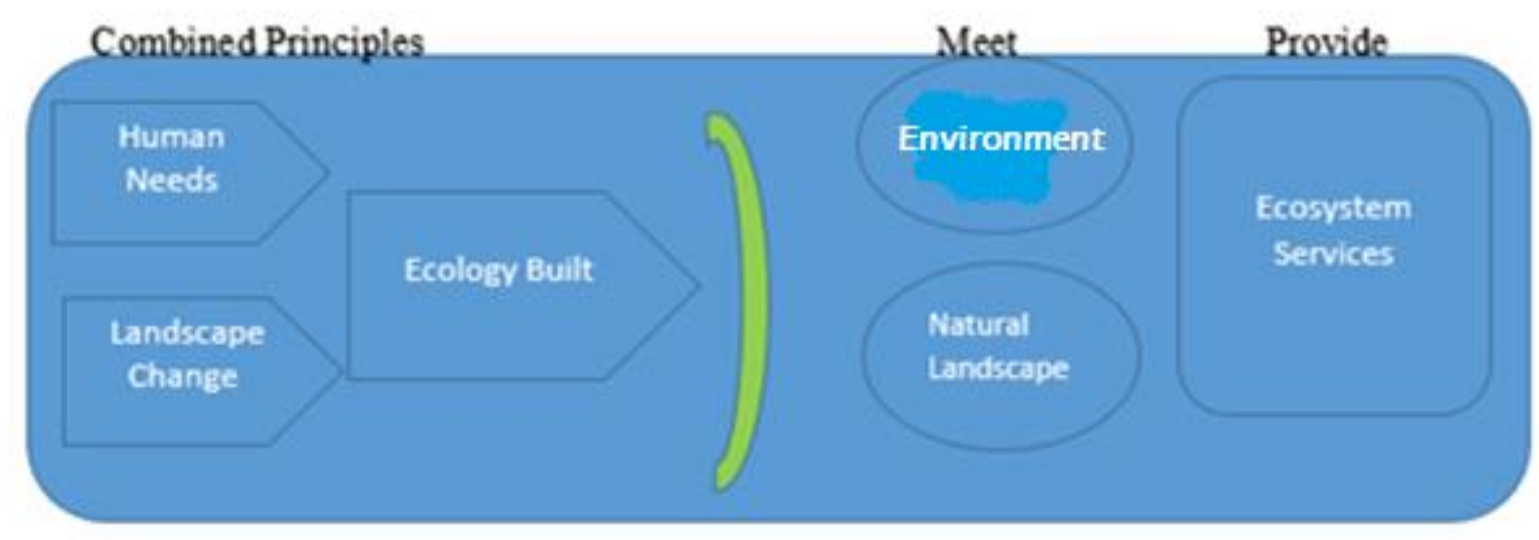

Fig. 2. Cycle of human ecology and environment Services.

\section{Conclusion}

Environment, landscape and nature are strongly interconnected each other and affect the human life in different stages in different time periods. The landscape changes are natural and modified by human to meet the needs. The relation between natural landscape and human environment can't identify by a single framework because it consists of a long chain form and deep structure. Finally the landscape changes are contained in different eras of time, sometime slow and sometime more accelerated, but gives us a lot of information about physical and cultural changes happening to them and help us to make policies and landscape management, the interrelationship between landscape change and human environment open door for further research.

\section{References}

Arntzen S (1982) Cultural landscape and Approaches to nature - Ecophilosophical Perspectives. 27-49. Bekoff GAB a M (2000) Integrating humans and nature: reconciling the boundaries of science and society. Elsevier Science Ltd, 15(8), 308-309. doi: 0169-5347

Çelik F (2013) Ecological Landscape Design. InTech. doi: 10.5772/55760

Clive A, McAlpine LMS, Tiffany HM and Rhodes a JR (2013) Strengthening Landscape Ecology's Contribution to a Sustainable Environment. Landscape Ecology for Sustainable Environment and Culture. doi: 10.1007/978-94-007-6530-6_2

Dani Belanche, 1. V. C., Carlos Orus. (2016). Cities. ELSEVIER, 50, 75-81.

El-Shafie DM (2010) Human-Environment Interactions: Phenomenal Relationships. International Journal of Civil \& Environmental Engineering, 10(4).

Erickson, CL (2010) The Transformation of Environment into Landscape: The Historical Ecology of Monumental Earthwork Construction in the Bolivian Amazon. Diversity, 618-652. doi: $10.3390 / \mathrm{d} 2040619$

Frederick Steiner LM (2017) observations, reflection, action. ELSEVIER.

Fuentes A and Baynes-Rock M (2017) Anthropogenic Landscapes, Human Action and the Process of Co-Construction with other Species: Making Anthromes in the Anthropocene. MDPI, 1-12. doi: 10.3390

Garkovich TG a L (1994) Landscape: The social Construction of nature and the enviroment. The Rural sociological Society 59, 1-24.

Kaymaz IC (2012) Landscape Perception. InTech, 251-277.

Landscapes of the Future (2011) The Parliamentary Office of Science and Technology.

Langellotto-Rhodaback SD a G (2015) Ecological Design of Urban Landscapes:

Economic, Social, and Ecological Benefts. Oregon State University.

Velardea MaD, Fryb G, Tveit M (2007) Health effects of viewing landscapes - Landscape types in 
environmental psychology. Elsevier, 199-212. doi: 10.1016/j.ufug.2007.07.001

Matthias Burgi AMH a NS (2004) Driving force for landscape change- current and new direction. Klawer Acedemic Publisher., 19(Land scape ecology), 857-868.

Mojtaba Parsaee PM a MS (2015) Investigating the Significance of landscape in Designing Library by Emphasizing on the Enhancement of Learning. Architectural Engineering Technology. doi: $10.4172 / 2168-9717.1000140$

Riley a PMu (2015) Sustainable Landscape Design. Oregon State University and University of Alaska Fairbanks(15), 275-295.

Roberta Aretano IP, Nicola Zaccarelli, Teodoro Semeraro, Giovanni Zurlini (2013) Landscape and Urban Planning. ELSEVIER, 112, 75-81.

Zube EH (1987) Perceived land use patterns and landscape values. SPB Academic Publishing, The Hague, 1(1), 37-45. 